\title{
Reproducing slugging oscillations of a real oil well
}

\author{
Florent Di Meglio, Glenn-Ole Kaasa, Nicolas Petit, Vidar Alstad
}

\begin{abstract}
This paper addresses the problem of reproducing oscillations generated by the well-known slugging phenomenon in multiphase flow. Reported investigations show how to determine the parameters of a recently proposed ordinary differential equations system, so that it captures the characteristics of actually observed slugging oscillations. A tuning procedure based on the mathematical properties of the model is presented. It is then applied to a test case consisting of a real oil well located in the North Sea. An observer using only topside measurements allows asymptotic reconstruction of critical variables such as the downhole pressure.
\end{abstract}

\section{INTRODUCTION}

For years, the slugging flow regime has been a concern for the oil industry. This phenomenon takes place in the long pipes connecting underground sources (reservoir or wellhead) to sub sea or surface production facilities. These pipes contain oil, water and gas forming a two-phase medium. Among all the possible two-phase flow regimes (bubbly, annular, churn, etc.) that can occur in this process of oil production, slugging is the less desirable one. In this regime, inhomogeneous spatial distribution of the liquid (usually oil and water) and gas phases coming from the sources generate oscillations of the flow rates and pressures inside the pipes, constituting an intermittent flow that is particularly visible at the outlet. These oscillations are referred to as the 'slugging' phenomenon, or slug flow. Their characteristics vary according to the pressure and temperature conditions, the geometry of the pipes or the composition of the gasliquid mixture. When their magnitude becomes too large, oil production must be stopped. A malicious effect is that, even when the production is not stopped, the oscillations reduce the overall oil production rate by increasing the average pressure in the pipes.

An important literature has been dedicated to the modeling of this phenomenon, in view of finding ways to suppress it. In the 1960s, hydrodynamic models [12], [7], [5] were developed to describe in details the characteristics (speed, length, frequency) of the 'slugs' of liquid traveling along the pipes. These models were meant to help predict the behavior of certain systems, by formulating necessary conditions for the existence of the slugs. Such conditions are

F. Di Meglio (corresponding author) is $\mathrm{PhD}$ candidate in Mathematics and Control at Centre Automatique et Systèmes, Unité Mathématiques et Syst'emes, MINES Paristech, 60, Bd St-Michel, 75272 Paris, Cedex 06, France

G.O. Kaasa is Research Engineer at Statoil ASA, Research Center Porsgrunn, Heroya Forksningspark 3908 Porsgrunn, Norway

N. Petit is Professor at Centre Automatique et Systèmes, Unité Mathématiques et Systèmes, MINES Paristech, 60, Bd St-Michel, 75272 Paris, Cedex 06, France

V. Alstad is Research Engineer at Statoil ASA, Research Center Porsgrunn, Heroya Forksningspark 3908 Porsgrunn, Norway presented in [13], and are based on the physical behavior of one slug ${ }^{1}$. In the 1980s, transient mechanistic models have been proposed. They were very soon embedded into commercial multiphase flow simulators, such as TACITE [8] and OLGA [1]. These simulation-oriented models are composed of nonlinear coupled sets of partial differential equations (PDE). Their steady-state behavior is very accurate when compared against experimental or even real field data, for certain classes of systems (in terms of size, pressures, velocities, etc.). They also predict with a relatively good accuracy the transient behavior of certain slugging systems, especially risers with low-points ${ }^{2}$. Yet, they are very difficult to analyze from a mathematical standpoint, besides trial anderror sensitivity analysis. The mere existence of solutions is by no means evident, let alone their stability.

More recently, simpler transient models have been developed for analytic studies and control design purposes. Jansen et al. describe in [11] the physical principles of a model further detailed in [10]. Their model, which is able to reproduce the slugging phenomenon for gas-lifted wells, is composed of three ordinary differential equations (ODE) with lumped states. They correspond to mass conversation laws for two volumes of gas, and one for the liquid. The relative simplicity of this model, compared to more involved PDE ones, allows for greater analysis possibilities. Sinègre [16] for example, has proven the existence of a periodic orbit for the model, under simplifying assumptions. More importantly, the model can be used to design control laws in view of suppressing the slugging phenomenon [9], [10], or estimators for unmeasured pressures [6], [14], [16]. Similarly, Storkaas et al. [18] have proposed a similar third order ODE model able to reproduce the behavior of slugging risers presenting a low-point. His study of the linear properties of the model gives insight into the controllability of the slugging phenomenon. Also, the model was used by Siahaan et al. [15] to propose a nonlinear control structure.

The downside of having such ODE models is that they may be too simple to reflect all the subtle characteristics of the slugging phenomenon. As a result, some of the parameters of the model need to be carefully tuned, in order

\footnotetext{
${ }^{1}$ Similarly, Bøe [2] and Taitel [19] derived, from force balance equations expressed on the body of one slug, conditions on the superficial velocities of gas and liquid to predict whether a given system will experience slugging or not. Much later, these criteria were proved to correspond to a Hopf bifurcation by Zakarian [20], who analyzed a simpler DAE model and showed that, when varying certain parameters, the stability properties of the system switch at a certain critical (bifurcation) point.

${ }^{2}$ Unfortunately, they fail to predict the instability of some systems (e.g. purely vertical wells), in the sense that, in certain cases, they may predict a steady flow regime for wells that are actually experiencing slugging.
} 
to allow the model to fit experimental data. Storkaas e.g. describes in [17] a tuning procedure used to find the values of five of the parameters of the model. This, of course, requires the a priori knowledge of data regarding the system under consideration. The upside is that, despite their relative simplicity, the models are able to compete with much more involved models, in terms of matching the behavior of real systems.

In this article, we show that the model presented in [3] can reproduce with a very good accuracy the behavior of a real well. This is the contribution of this article. In a first step, the parameters of the model are estimated by simple tuning rules based on the physical properties and a mathematical analysis of the model. Then, an observer is designed to reconstruct unmeasured states from topside pressure measurements. These results must be put into perspective of the stabilization results presented in [4]. There, the proposed model-based controller was able to outperform PI controllers when tested on an experimental multiphase flow loop, which is a down-scaled version of a real system. The ability of the model to match the behavior of a real well, and to improve upon the performances of PI controllers on experimental facilities, suggest that a control design based on it has some potential to stabilize the flow on actual slugging wells.

The paper is organized as follows. In Section II, we review the physical principles and the equations of the model proposed in [3]. Then, in Section III, we introduce the tuning rules and list the information needed. Then, in Section IV, we focus on the design of an observer. It is experimentally validated on real-well data. We give some conclusions and perspective in Section V.

\section{REVIEW OF THE MODEL}

Consider the pipe schematically depicted in Figure 1. It is filled with gas and liquid inflows from the reservoir at constant rates. The outflow can be regulated by a valve (production choke). The model proposed in [3] stresses the possible existence of an obstructing liquid interface restricting the flow of gas, modeled as a virtual valve. In the case of a pipe presenting a low-point angle, this precisely corresponds to the idea originally proposed in the Storkaas model [18], where the height of liquid at the low-point determines the opening of the virtual valve. The main modeling assumption in [3] consists in considering the existence of such a valve, even when no low-point exists. The virtual valve separates the pipe into two parts. Upstream the valve, the existence of an elongated bubble of gas, of constant volume $V_{e b}$, is assumed. The section downstream the valve is referred to as the riser. It has a length $L$, and the flow there is assumed to be distributed. Only a fraction of the gas (determined by $0<\epsilon<1$ ) accumulates in the elongated bubble, causing a build-up of pressure at the roots of the instability. When the pressure in the bubble gets high enough, gas flows through the virtual valve into the riser. This increases the pressure there, and pushes the liquid out of the system. In this model, the state variables are the mass

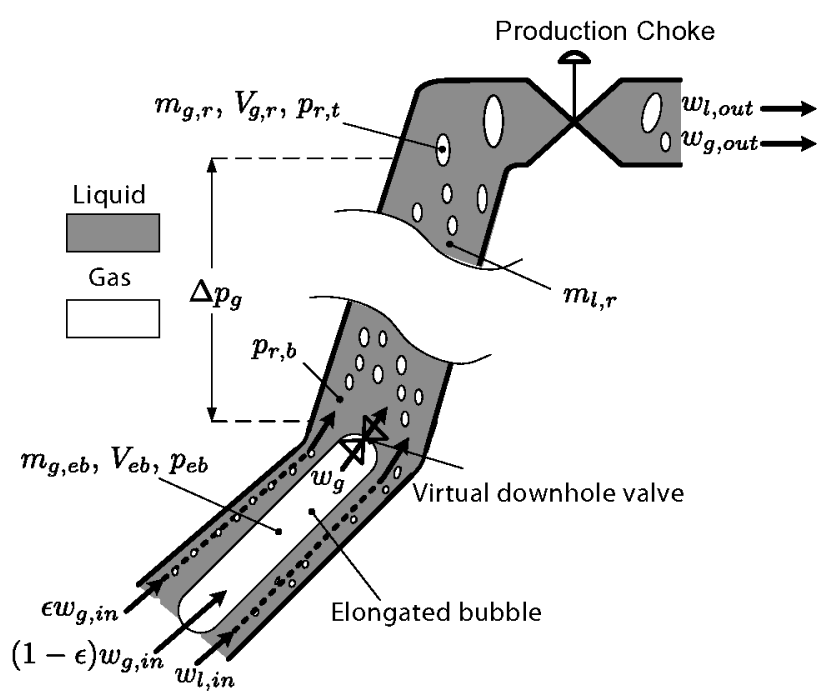

Fig. 1. Schematic view of an inclined riser carrying an inhomogeneous blend of liquid and gas

of gas in the elongated bubble $m_{g, e b}$, the mass of gas in the riser $m_{g, r}$, and the mass of liquid in the riser $m_{l, r}$. The first principle equations read

$$
\begin{aligned}
\dot{m}_{g, e b}(t) & =(1-\epsilon) w_{g, \text { in }}-w_{g}(t) \\
\dot{m}_{g, r}(t) & =\epsilon w_{g, \text { in }}+w_{g}(t)-w_{g, \text { out }}(t) \\
\dot{m}_{l, r}(t) & =w_{l, \text { in }}-w_{l, \text { out }}(t)
\end{aligned}
$$

where the $g$ and $l$ subscripts stand for gas and liquid respectively, in and out refer to inflow and outflow, and $w_{g}(t)$ is the flow of gas through the virtual valve. Apart from the inflows, which are assumed constant, the flow rates depend on the pressure drop over the choke under consideration. A linear relation of the form

$$
w_{g}=C_{g} \max \left(0, p_{e b}-p_{r, b}\right)
$$

where $p_{r, b}$ is the pressure at the bottom of the riser, is chosen for the virtual valve. The $\max (0, \cdot)$ function guarantees that no back flow can be admitted through the valve. The flow through the production choke, which is diphasic, requires a more accurate relation given by

$$
w_{\text {out }}=u C_{c} \sqrt{p_{r, t}-p_{s}}
$$

where $u \in(0,1)$ is the controlled opening of the choke, $p_{r, t}$ and $p_{s}$ the pressure upstream and downstream the choke, respectively. The dependence with respect to density is neglected, assuming that the mixture density at the choke is constant. Then, the flows of gas and liquid through the production choke are given by their mass fractions, which yields

$$
\begin{aligned}
w_{l, \text { out }} & =\frac{m_{l, r}}{m_{l, r}+m_{g, r}} w_{\text {out }} \approx w_{\text {out }} \\
w_{g, \text { out }} & =\frac{m_{g, r}}{m_{l, r}+m_{g, r}} w_{\text {out }} \approx \frac{m_{g, r}}{m_{l, r}} w_{\text {out }}
\end{aligned}
$$

Finally, the pressures (topside, and in the elongated bubble) are given by the ideal gas law, under isothermic assumptions. 
The volume of the elongated bubble $V_{e b}$ is assumed to be constant, whereas the gas downstream the virtual choke is assumed to be compressible. Its volume $V_{g, r}$ depends on the mass of liquid in this part

$$
V_{g, r}=L A-\frac{m_{l, r}+m_{l, s t i l l}}{\rho_{l}}
$$

where $L$ is the length of the riser and $A$ the cross-section area. $m_{l, \text { still }}$ represents the mass of liquid present at all times in the riser, which is not concerned by the dynamics. Besides, the pressure drop over the whole riser is assumed to be gravitydominated (friction being neglected)

$$
\begin{aligned}
& p_{e b}=\frac{R T}{M V_{e b}} m_{g, e b} \\
& p_{r, b}=p_{r, t}+\frac{g \sin \theta}{A}\left(m_{l, r}+m_{l, s t i l l}\right) \\
& p_{r, t}=\frac{R T}{M\left(L A-\frac{m_{l, r}+m_{l, s i l l}}{\rho_{l}}\right)} m_{g, r}
\end{aligned}
$$

where $\theta$ is the mean inclination of the pipe.

\section{TUNING PROCEDURE}

In this section, we describe how to choose the parameters in model (1)-(2)-(3) to match the behavior of a given system. These parameters are: $L, \theta, A, g, \rho_{l}, R, T, M, p_{s}, w_{g, i n}$, $w_{l, i n}, C_{c}, m_{l, s t i l l}, V_{e b}$ and $\epsilon$. The procedure requires a priori information on the system. First, the geometry of the pipe is required to set the values of $L, A$, and $\theta$. The equilibrium values of the topside pressure $\bar{y}_{r, t}(\bar{u})$ and downhole pressure $\bar{y}_{r, b}(\bar{u})$ at one operating point $\bar{u}$ are also required. These values can be measured, when corresponding sensors are available, or otherwise estimated by an advanced multiphase flow simulator (such as $\mathrm{OLGA}^{\mathrm{TM}}$ ). They determine the correct values of the choke constant $C_{c}$ and the still mass of liquid $m_{l, s t i l l}$. Also, the value of the production choke opening that causes oscillations to appear on the real system $u^{*}$ is needed. The analytic study of the bifurcation point of the model then determines the volume $V_{e b}$ of the elongated bubble. Eventually, the magnitude of the pressure oscillations determines the fraction of gas $\epsilon$ that is not trapped in the elongated bubble.

\section{A. Length of the riser}

The riser is defined, in the model, as the part of the pipe downstream the virtual valve. The location of this valve is unknown a priori. In some cases, the geometry suggests a natural location, where an irregularity in the flow is likely to occur. It usually takes the form of an angle, when the well has a near-horizontal part followed by an inclined one. Once again, in the case of a low-point angle (when the near-horizontal part is declining), this assumption is consistent with the works of Storkaas [17]. When nothing in the geometry suggests the existence of an irregularity, other considerations, such as the location of the sensors can determine the most suited location for the virtual valve. The geometry of the real well considered in this article, depicted on figure 5, clearly indicates that the virtual valve must be placed at the end of the near-horizontal section. This location coincides with that of the downhole pressure sensor.

\section{B. Straightforward parameters}

Numerous parameters in the model can be directly inferred from the geometry of the system, or the nature of the liquidgas mixture. For example, the inclination parameter $\theta$ is the mean inclination of the pipe, and the cross-section area $A$ is known. Similarly, the separator pressure and the temperature inside the pipe are measured and almost constant in practice. As a result, values for $\theta, A, g, \rho_{l}, R, T, M$ and $p_{s}$ can be obtained from the geometry of the well under consideration and PVT data sheets for the fluid characteristics.

\section{Inflow rates}

Next, values must be given to the inflow rates of liquid $\left(w_{l, \text { in }}\right)$ and gas $\left(w_{g, \text { in }}\right)$. The difficulty is that they are scarcely measured. Besides, they are not constant: the assumption that they are is a simplification, which is formulated to ease the computations. We use in the model the values of the time-average outflow rates, commonly measured by Multiphase Flow Meters (MPM). At steady-state, the inflow and outflow rates must be constant and equal. Even though the average values of the outflow rates do not match their equilibrium values ${ }^{3}$, they lie in the same range. This reveals accurate enough for the model to reproduce the behavior of the original system. When no flow meter can be used, an alternative solution is to use a commercial multiphase flow simulator like $\mathrm{OLGA}^{\mathrm{TM}}$ to compute the steady-state values of the inflow rates from the system characteristics.

\section{Choke constants}

As previously mentioned, the model contains two valve equations (4)-(5): one for the virtual valve, and one for the production valve. The choke constant for the production valve is usually provided by its manufacturer. Yet, equation (5) assumes that the density of the mixture is constant. Therefore, the equilibrium value of the topside pressure cannot perfectly fit the system for all values of the choke opening. For our model, it is given by $\bar{p}_{r, t}=p_{s}+\frac{w_{l, i n}^{2}}{\rho_{l} C_{c}^{2} u^{2}}$. In order to find a for $C_{c}$, we choose to match it with the one of the real system $\bar{y}_{r, t}(\bar{u})$ for one value of the choke opening $\bar{u}$. To measure the equilibrium topside pressure $y_{r, t}(\bar{u}), \bar{u}$ has to lie in the stable region $\bar{u}<u^{*}$. On the other hand, the chosen equilibrium of the model should be as close as possible to the real one in the unstable region, where the flow must be stabilized. As a result, $\bar{u}$ is taken as close as possible to the bifurcation point, but strictly in the stable region. The value of $C_{c}$ must be chosen so that

$$
C_{c}=\frac{w_{l, i n}}{\bar{u} \sqrt{\bar{y}_{r, t}-p_{s}}}
$$

The second choke constant appearing in (4) is that of the virtual valve. Because it is difficult to mathematically study its impact on the dynamics, a rough estimate has to be picked manually at this stage of the tuning procedure. It may then be

\footnotetext{
${ }^{3}$ which is precisely the reason why it is desired to stabilize the flow
} 
adjusted afterwards, once the parameters have been chosen, to slightly reshape the simulated oscillations. A typical value for this parameter is $C_{g}=10^{-4} \mathrm{~kg} . \mathrm{s} \cdot \mathrm{m}^{-1}$.

\section{E. Still mass of liquid}

As mentioned in Section II, the parameter $m_{l, s t i l l}$ represents the (minimum) mass of liquid present in the riser at all times. Its value is chosen using the equilibrium value of the bottom hole pressure for one operating point $\bar{u}$, given by

$$
\begin{aligned}
\bar{p}_{r, b}(\bar{u}) & =\bar{p}_{r, t}(\bar{u})+\left(\bar{m}_{l, r}(\bar{u})+m_{l, s t i l l}\right) \frac{g \sin \theta}{A} \\
& =\bar{p}_{r, t}(\bar{u})+\left(\frac{\bar{p}_{r, t}(\bar{u})}{\bar{p}_{r, t}(\bar{u})+b \bar{x}}\left(\rho_{l} L A-m_{l, s t i l l}\right)+m_{l, s t i l l}\right) \frac{g \sin \theta}{A} \\
& =\bar{p}_{r, t}(\bar{u})+\frac{\bar{p}_{r, t}(\bar{u}) L A \rho_{l}+b \bar{x} m_{l, s t i l l}}{\bar{p}_{r, t}(\bar{u})+b \bar{x}} \frac{g \sin \theta}{A}
\end{aligned}
$$

where $\bar{x}=\frac{w_{g, i n}}{w_{l, i n}}$ is the equilibrium gas-liquid mass ratio, and $b=\frac{\rho_{l} R T}{M}$. All the parameters except $m_{l, s t i l l}$ in this equation are known. Therefore, knowing (or, once again, estimating it with $\mathrm{OLGA}^{\mathrm{TM}}$ ) the value of the steady-state bottom hole pressure $\bar{y}_{r, b}(\bar{u})$ of the system for one operating point $\bar{u}$, one should set the parameter $m_{l, s t i l l}$ to

$$
m_{l, s t i l l}=\frac{\bar{y}_{r, b}-\bar{y}_{r, t}}{g / A \sin \theta}\left(1+\frac{\bar{y}_{r, t}}{b \bar{x}}\right)-\frac{\bar{y}_{r, t}}{b \bar{x}} V_{r} \rho_{l}
$$

The value of $m_{l, s t i l l}$ highly depends on the system under consideration: it can be 0 , e.g. for short flowline risers, and can reach $\frac{1}{2} V_{r} \rho_{l}$, which corresponds to at least half the riser being permanently filled with liquid.

\section{F. Elongated bubble}

A value for the volume of the elongated bubble $V_{e b}$ has yet to be determined. This parameter is used to set the position of the bifurcation point of the model to that of the real system, which we note $u^{*}$. More specifically, the bifurcation point is characterized by the appearance of two purely imaginary eigenvalues. To find the best value for $V_{e b}$, one could write Routh's criterion for the Jacobian matrix $J\left(u^{*}\right)$ (expressions for the Jacobian matrix coefficients are reported in Appendix), and solve the obtained equation with respect to $V_{e b}$. Unfortunately, the criterion does not take the form of an analytically tractable expression, and $V_{e b}$ must be determined with a numerical solver (e.g. Newton's method). One can achieve this by solving the following problem

$$
\mathfrak{R}\left\{\lambda\left(u^{*}, V_{e b}\right)\right\}=0
$$

with respect to $V_{e b}$, where $\lambda$ is one of the two complex conjugate eigenvalues of the system (the third eigenvalue being always real). Figure 2 represents the variation, for $u=$ $u^{*}$, of the real part of $\lambda$ as $V_{e b}$ increases. The corresponding locus of the eigenvalues (parametrized by $V_{e b}$ ) are plotted in Figure 3.

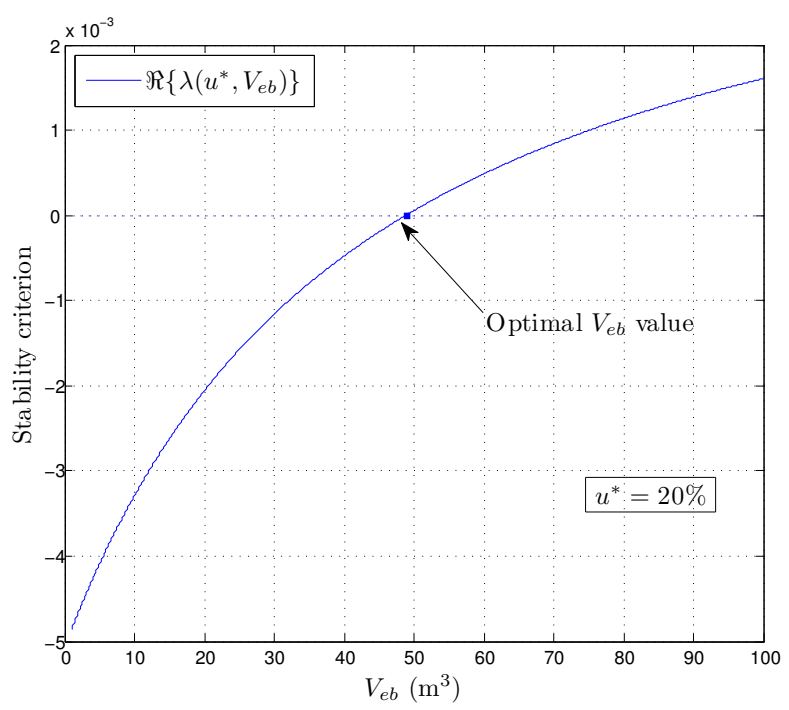

Fig. 2. Value of $\mathfrak{R}\left\{\lambda\left(u^{*}, V_{e b}\right)\right\}$ as $V_{e b}$ increases. When the eigenvalues cross the imaginary axis, the criterion is exactly 0 . The corresponding value of $V_{e b}$ should be used in the model.

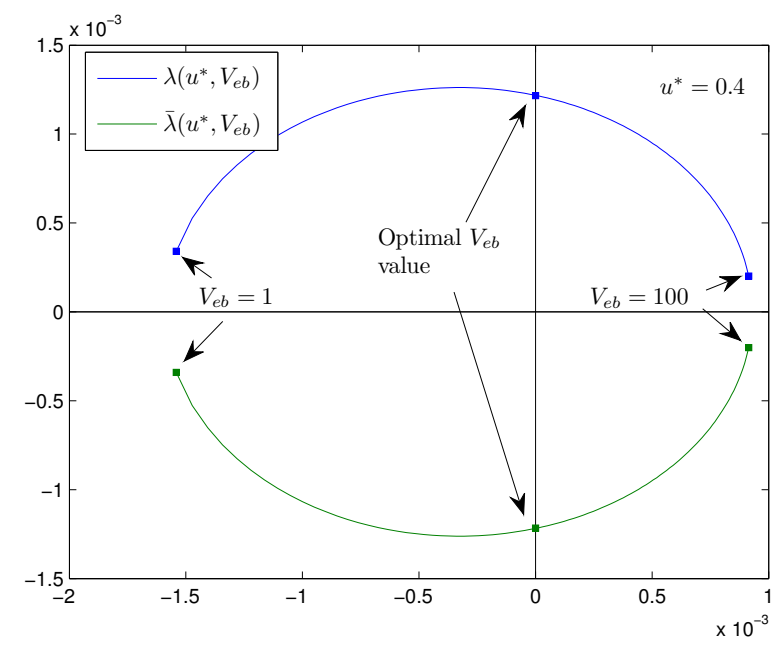

Fig. 3. Locus of the eigenvalues $\lambda\left(u^{*}\right)$ and $\bar{\lambda}\left(u^{*}\right)$ when $V_{e b}$ varies. For low values of $V_{e b}$, the eigenvalues are in the LHP. As the parameter increases, the eigenvalues cross the imaginary axis, which corresponds to the criterion plotted in Figure 2 being 0.

\section{G. Split inflow of gas}

Eventually, one must choose a value for $\epsilon$, which determines the fraction of the amount of gas that accumulates into the elongated bubble. $\epsilon$ directly impacts on the magnitude of the pressure oscillations. In particular, it can be mathematically proven ${ }^{4}$ that when the virtual valve is closed, i.e. when $p_{e b}<p_{r, b}$, the mass of liquid $m_{l, r}$ converges asymptotically to $\underline{m}_{l, r}=\frac{\bar{p}_{r, t}}{\bar{p}_{r, t}+\epsilon b \bar{x}}\left(\rho_{l} L A-m_{l, s t i l l}\right)$, and the topside pressure converges to $\bar{p}_{r, t}$. Yet, these values are only approached because the virtual valve does not remain closed in the observed oscillations. Therefore, the pressure at the base of

\footnotetext{
${ }^{4}$ thanks to an analytic integration of (1)-(2) and its asymptotics.
} 
the riser $p_{r, b}$ converges to a "pseudo-equilibrium"

$$
\underline{p}_{r, b}=\bar{p}_{r, t}+\left(\underline{m}_{l, r}+m_{l, s t i l l}\right) \frac{g \sin \theta}{A}
$$

When the virtual valve opens again, the mass of liquid soon drops. This point is illustrated in Figure 4. Therefore, $\underline{p}_{r, b}$ is

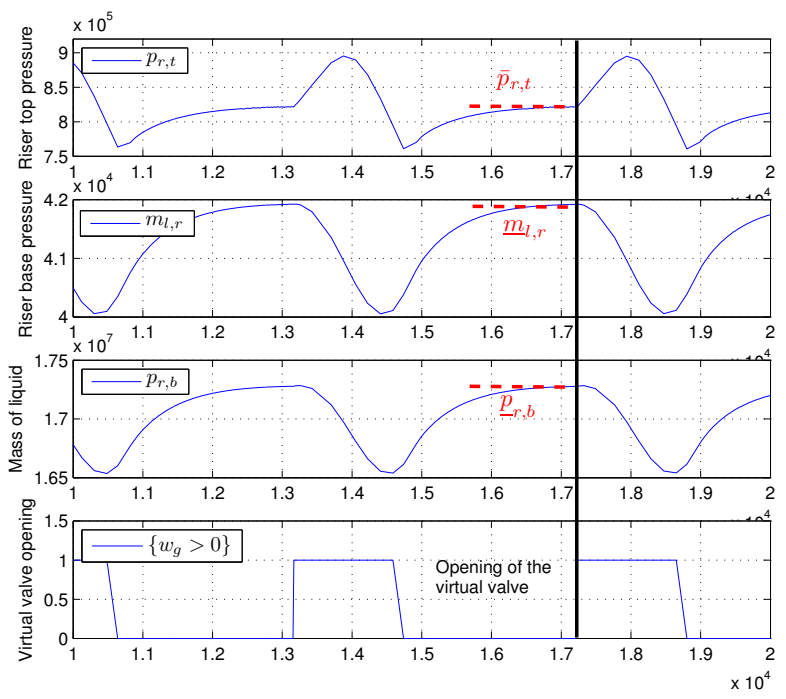

Fig. 4. Convergence to a pseudo-equilibrium depending on $\epsilon$

a good approximation of the maximum riser base pressure over the cycle. When a measure of the bottom hole pressure is available, $\epsilon$ can be chosen to match the peak of the oscillations of $p_{r, b}$ with that of the system $\bar{y}_{r, b}^{\max }$ by setting

$$
\epsilon=\frac{\bar{y}_{r, t}}{b \bar{x}} \frac{\rho_{l} V_{r}-\left(y_{r, b}^{\max }-\bar{y}_{r, t}\right) \frac{A}{g \sin \theta}}{\left(y_{r, b}^{\max }-\bar{y}_{r, t}\right) \frac{A}{g \sin \theta}-m_{l, s t i l l}}
$$

Otherwise, when no measure of the downhole pressure is available, $\epsilon$ has to be tuned manually, with a trial-and-error approach to match the magnitude of the topside pressure oscillations. As indicated by the expression of $\underline{m}_{l, r}$, the magnitude of the oscillations is a decreasing function of $\epsilon$.

\section{H. Summary}

Table I gives a summary of the tuning procedure, along with the corresponding values for the case we study here. These values, reproduced courtesy of Statoil, correspond to an actual 7731 m-long well in the North Sea, schematically depicted on Figure 5. As mentioned in Section III-A, the location of the virtual valve coincides with that of the downhole pressure sensor, at the end of the near-horizontal section. This assumption is validated by the resultant value of volume $V_{e b}=48 \mathrm{~m}^{3}$. Indeed, the total volume of the pipe is, in the model, $L A+V_{e b}=140.8 \mathrm{~m}^{3}$, which is very close to the actual total volume of the well $V=140.4 \mathrm{~m}^{3}$. One should notice that nothing, in the computation of $V_{e b}$, guarantees this equality a priori. In general, it would be possible to obtain a total volume $L A+V_{e b}$ greater or smaller than the total well volume. A greater volume would correspond to an elongated bubble originating upstream the well (in the case

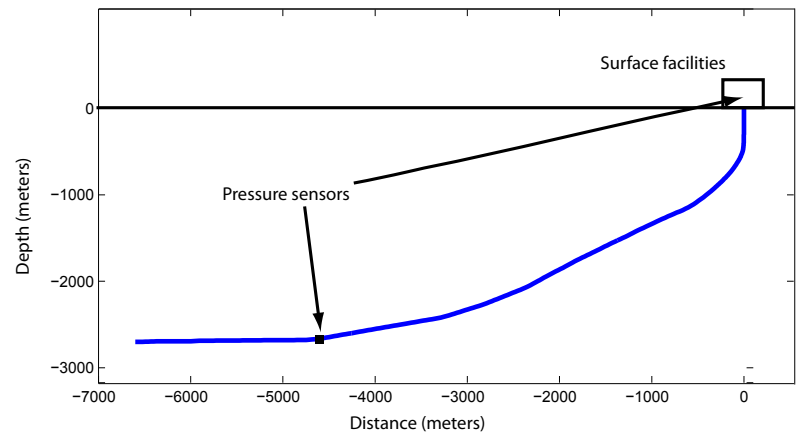

Fig. 5. Schematic view of the considered well

of a reservoir, e.g.). A smaller value implies that the gas pocket occupies only a part of the volume of the well-section upstream the riser. This is also a valid assumption. Too large or inconsistent discrepancies should lead to reconsider the candidate value for $L$ at the beginning of the identification process.

\begin{tabular}{|c|c|l|}
\hline Variable & Information used & Value \\
\hline$R$ & - & $R=8.314 \mathrm{~J}^{-1} \mathrm{~mol}^{-1}$ \\
\hline$g$ & - & $g=9.81 \mathrm{~m} \cdot \mathrm{s}^{-2}$ \\
\hline$T$ & Topside temperature sensor & $T=363 \mathrm{~K}$ \\
\hline$L$ & Geometry of the well & $L=5200 \mathrm{~m}$ \\
\hline$A$ & Geometry of the well & $A=1.77 \times 10^{-2} \mathrm{~m}^{2}$ \\
\hline$\theta$ & Geometry of the well & $\theta=0.78 \mathrm{rad}$ \\
\hline$\rho_{l}$ & PVT data sheet & $\rho_{l}=900 \mathrm{~kg} \cdot \mathrm{m}^{-3}$ \\
\hline$M$ & PVT data sheet & $M=22 \times 10^{-3} \mathrm{~kg} \cdot \mathrm{mol}^{-1}$ \\
\hline$p_{s}$ & Separator pressure sensor & $p_{s}=6.6 \mathrm{bar}$ \\
\hline$w_{l, i n}$ & Multiphase flow meter & $w_{l, i n}=11.75 \mathrm{~kg} \cdot \mathrm{s}^{-1}$ \\
\hline$w_{g, i n}$ & Multiphase flow meter & $w_{g, i n}=0.82 \mathrm{~kg} \cdot \mathrm{s}^{-1}$ \\
\hline$C_{c}$ & $\begin{array}{c}\text { Equilibrium topside pressure } \\
\text { at } \bar{u}=35 \%: \bar{y}_{r, t}=8.23 \text { bar }\end{array}$ & $C_{c}=8.32 \times 10^{-2} \mathrm{~m}$ \\
\hline$m_{l, s t i l l}$ & $\begin{array}{c}\text { Equilibrium downhole pressure } \\
\text { at } \bar{u}=35 \%: \bar{y}_{r, b}=170 \text { bar }\end{array}$ & $m_{l, s t i l l}=3.73 \times 10^{4} \mathrm{~kg}$ \\
\hline$V_{e b}$ & Bifurcation point $u^{*} \approx 20 \%$ & $V_{e b}=48 \mathrm{~m}^{3}$ \\
\hline$\epsilon$ & $\begin{array}{c}\text { Max value of the downhole } \\
\text { pressure } \bar{y}_{r, b}^{\text {max }}=176 \text { bar }\end{array}$ & $\epsilon=0.78$ \\
\hline
\end{tabular}

TABLE I

LIST OF PARAMETERS USED IN THE MODEL

\section{RESUlting DYNAMICS AND OBSERVER DESIGN}

Figure 6 shows a comparison between the model dynamics resulting from the aforedescribed tuning procedure and actual well measurements. Because the length $L$ coincides with the depth of the sensor, the reconstructed downhole pressure and the measurements can be directly compared. Should this length differ from the sensor depth, some compensation would have been considered, to provide fair comparisons. The results show the relevance of the model as the magnitude and frequency of the simulated oscillations almost match that of the real system. One should notice the scarce sampling rate of the downhole pressure measurements. This is frequent with downhole sensors which are often unreliable, tend to deviate and sometimes break down due to the harshness of their environment. We now show how to use the model in an 

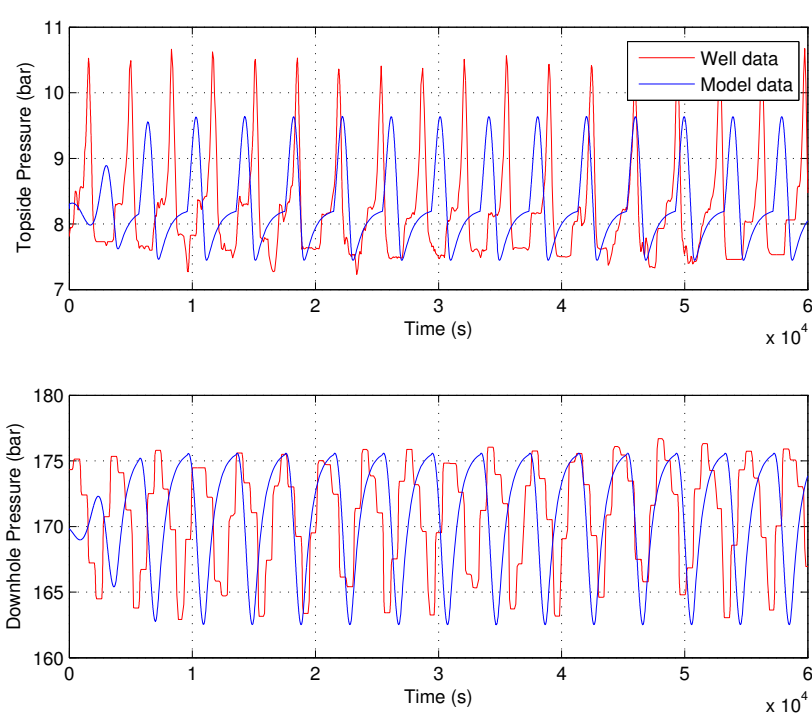

Fig. 6. Topside and downhole pressure oscillations. Comparison between the (open-loop) model and well dynamics at $u=35 \%$.

observer structure to synchronize it with the actual system, and reconstruct a robust estimate of the downhole pressure.

\section{A. Observer design}

In this part, we consider the problem of estimating the whole state of system (1)-(2)-(3) when only the topside pressure $p_{r, t}$ is measured. This is an important topic from an engineering point of view, because this measurement is often available. First, we re-write the model in the new set of coordinates $x=\left(x_{1}, x_{2}, x_{3}\right)=\left(m_{g, e b}, p_{r, t}, m_{l, r}\right)$

$$
\begin{aligned}
\dot{x}_{1} & =f_{1}(x) \\
& =(1-\epsilon) w_{g, \text { in }}-C_{g} \max \left(0, a x_{1}-x_{2}-c\left(x_{3}+m_{3, \text { still }}\right)\right) \\
\dot{x_{2}} & =f_{2}(x) \\
& =\frac{b}{m_{3}^{\Delta}-x_{3}}\left[\epsilon w_{g, \text { in }}+C_{g} \max \left(0, a x_{1}-x_{2}-c\left(x_{3}+m_{l, s t i l l}\right)\right)\right. \\
& \left.+w_{l, i n} \frac{x_{2}}{b}-\frac{m_{3}^{\Delta}}{x_{3}} \frac{x_{2}}{b} u C_{c} \sqrt{\bar{\rho}_{m}\left(x_{2}-p_{s}\right)}\right] \\
\dot{x_{3}} & =f_{3}(x) \\
& =w_{l, \text { in }}-u C_{c} \sqrt{\bar{\rho}_{m}\left(x_{2}-p_{s}\right)}
\end{aligned}
$$

where $a=\frac{R T}{M V_{e b}}$ and $c=g / A \sin \theta$. The measured output is now $p_{r, t}=x_{2}$. The observer equations read

$$
\begin{aligned}
& \dot{\hat{x}}_{1}=f_{1}(\hat{x}) \\
& \dot{\hat{x}}_{2}=f_{2}(\hat{x})-k\left(\hat{x}_{2}-x_{2}\right) \\
& \dot{\hat{x}}_{3}=f_{3}(\hat{x})
\end{aligned}
$$

where $k$ is the observer gain. Even though this resembles a high-gain structure, we were not able to come up with a proof of the convergence yet. This point will be the subject of future investigation. We only present here an experimental validation of this approach.

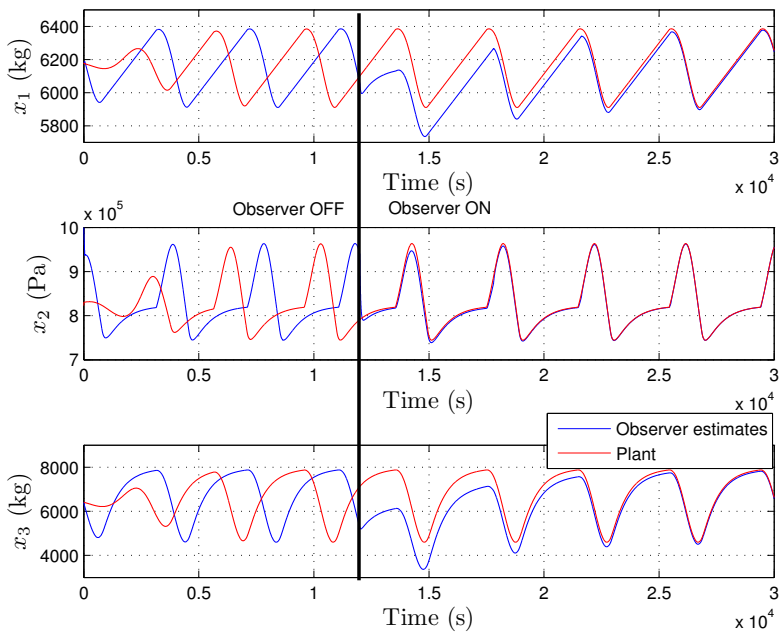

Fig. 7. Convergence of the observer to the model trajectory. The observer only needs one oscillation to be synchronized with the plant, and a few more to converge completely.

\section{B. Simulations and experimental validation}

a) Simulation results: The observer was first tested in simulation, on the model itself. Figure 7 shows the convergence of the trajectory of the observer to that of the plant. The observer was turned on $12000 \mathrm{~s}$ after the start of the simulation.

b) Real well data: The observer was then tested against real measurements coming from the above mentioned well. Figure 8 shows a comparison between the measured pressures and the estimates computed by the observer using only the topside pressure measurement. Not only this method allows to synchronize the model with the system, but it also provides a good estimation of the downhole pressure. As presented in [4], these estimates can then be used in a control algorithm to stabilize the flow and suppress the slugging phenomenon. In particular, the estimated downhole pressure can replace the measurements, compensating for their large sampling time.

\section{Conclusion}

A method to tune the parameters of the model presented in [3] was proposed. The method is based on physical considerations and a mathematical analysis of the model. The efficiency of this technique is illustrated by comparison with real slugging well data. It highlights the relevance of the model itself and its capability to reproduce the behavior of slugging wells. An observer structure, allowing to reconstruct unavailable or unreliable pressure measurements has been presented, along with its experimental validation. As presented in [4], a controller can be derived from the model and allow suppression of the slugging phenomenon. The controller was tested on an experimental multiphase flow loop in Porsgrunn, Norway, with good success. In particular, it was able to stabilize the flow around relatively high values of the choke opening. Transposed on an actual offshore well, this result is expected to yield significant production increases. 

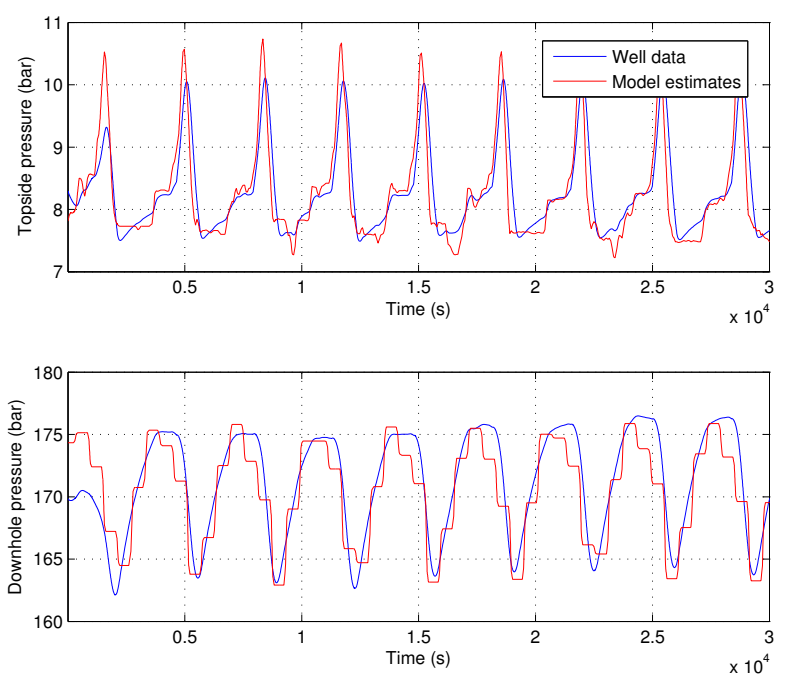

Fig. 8. Comparison between the observer estimates for $k=0.05$ and the real measurements. After only one oscillation, the system is perfectly synchronized in terms of frequency and amplitude. The observer allows to reconstruct the downhole pressure from topside measurements.

\section{REFERENCES}

[1] K. Bendiksen, D. Maines, R. Moe, and S. Nuland. The dynamic twofluid model olga: Theory and application. SPE production engineering, 6(2):171-180, 1991.

[2] A. Bøe. Severe slugging characteristics ; part i:flow regime for severe slugging ; part ii: Point model simulation study. Presented at Selected Topics in Two-Phase Flow, NTH, Trondheim, Norway, 1981.

[3] F. Di Meglio, G.-O. Kaasa, and N. Petit. A first principle model for multiphase slugging flow in vertical risers. Conference on Decision and Control, 2009.

[4] F. Di Meglio, G.-O. Kaasa, N. Petit, and V. Alstad. Model-based control of slugging flow: an experimental case study. 2010 American Control Conference (To appear), 2010.

[5] A. E. Dukler and M. G. Hubbard. A model for gas-liquid slug flow in horizontal and near horizontal tubes. Industrial $\mathcal{E}$ Engineering Chemistry Fundamentals, 14(4):337-347, 1975.

[6] G. O. Eikrem, B. Foss, L. Imsland, B. Hu, and M. Golan. Stabilization of gas lifted wells. Proceedings of the 15th IFAC World Congress, 15, Part 1, 2002.

[7] R. C. Fernandes, R. Semiat, and A. E. Dukler. Hydrodynamic model for gas-liquid slug flow in vertical tubes. AIChE Journal, 29:981-989, 1983.

[8] D. Ferre, V. Bouvier, and C. Pauchon. TACITE Physical Model Description Manual. Rapport IFP, 1995.

[9] K. Havre and M. Dalsmo. Active feedback control as the solution to severe slugging. SPE Annual Technical Conference, 2001.

[10] L. S. Imsland. Output Feedback and Stabilization and Control of Positive Systems. PhD thesis, Norwegian University of Science and Technology, Department of Engineering Cybernetics, 2002.

[11] B. Jansen, M. Daslmo, L. Nøkleberg, K. Havre, V. Kristiansen, and P. Lemetayer. Automatic control of unstable gas lifted wells. SPE annual technical conference, 1999.

[12] E.S. Kordyban. A flow model for two-phase slug flow in horizontal tubes. Trans. ASME, J. Basic Eng, 83:613-618, 1961.

[13] Z. Ruder, PJ Hanratty, and TJ Hanratty. Necessary conditions for the existence of stable slugs. International journal of multiphase flow, 15(2):209-226, 1989.

[14] F. Scibilia, M. Hovd, and R. R. Bitmead. Stabilization of gas-lift oil wells using topside measurements. Proceedings of the 17th IFAC World Congress, pages 13907-13912, 2008.

[15] H. B. Siahaan, O. M. Aamo, and Foss B. A. Suppressing riser-based slugging in multiphase flow by state feedback. Proceedings of the 44th Conference on Decision and Control, pages 12-15, 2005.

[16] L. Sinègre. Dynamic study of unstable phenomena stepping in gas-lift activated systems. PhD thesis, Ecole des Mines de Paris, 2006.
[17] E. Storkaas. Control solutions to avoid slug flow in pipeline-riser systems. PhD thesis, Norwegian University of Science and Technology, 2005.

[18] E. Storkaas and S. Skogestad. Stabilization of severe slugging based on a low-dimensional nonlinear model. AIChE Journal, 2002.

[19] Y. Taitel. Stability of severe slugging. International Journal of Multiphase Flow, 12(2):203 - 217, 1986.

[20] E. Zakarian. Analysis of two-phase flow instablilities in pipe-riser systems. Proceedings of Pressure Vessels and Piping Conference, 2000.

\section{APPENDIX}

The equations linearized around an operating point $u$ read $\frac{d}{d t}\left(m_{g, e b}, m_{g, r}, m_{l, r}\right)^{T}=J(u)\left(m_{g, e b}, m_{g, r}, m_{l, r}\right)^{T}$, with

$$
\begin{aligned}
J(1,1)= & -C_{g} \frac{R T}{M V_{e b}} \\
J(1,2)= & C_{g} \frac{\bar{p}_{r, t}(u)+b \bar{x}}{\bar{x} m_{l}^{\Delta}} \\
J(1,3)= & C_{g} \frac{\bar{p}_{r, t}(u)+b \bar{x}}{\bar{x} m_{l}^{\Delta}} \frac{\bar{p}_{r, t}(u)}{b}+C_{g} \frac{g \sin \theta}{A} \\
J(2,1)= & C_{g} \frac{R T}{M V_{e b}} \\
J(2,2)= & \frac{\bar{p}_{r, t}(u)+b \bar{x}}{m_{l}^{\Delta}}\left(-\frac{C_{g}}{\bar{x}}-\frac{w_{l, i n}}{\bar{p}_{r, t}(u)}-\frac{w_{l, i n}}{2\left(\bar{p}_{r, t}(u)-p_{s}\right)}\right) \\
J(2,3)= & \frac{\bar{p}_{r, t}(u)+b \bar{x}}{m_{l}^{\Delta}}\left(-\frac{C_{g}}{\bar{x}} \frac{\bar{p}_{r, t}(u)}{b}+\bar{x} \frac{w_{l, i n}}{\bar{p}_{r, t}(u)}\right. \\
& \left.-\frac{w_{l, i n}}{2\left(\bar{p}_{r, t}(u)-p_{s}\right)} \frac{\bar{p}_{r, t}(u)}{b}\right)-C_{g} \frac{g \sin \theta}{A} \\
J(3,1)= & 0 \\
J(3,2)= & -\frac{w_{l, i n}}{2\left(\bar{p}_{r, t}(u)-p_{s}\right)} \frac{\bar{p}_{r, t}(u)+b \bar{x}}{\bar{x} m_{l}^{\Delta}} \\
J(3,3)= & -\frac{w_{l, i n}}{2\left(\bar{p}_{r, t}(u)-p_{s}\right)} \frac{\bar{p}_{r, t}(u)+b \bar{x}}{\bar{x} m_{l}^{\Delta}} \frac{\bar{p}_{r, t}(u)}{b}
\end{aligned}
$$

where $m_{3}^{\Delta}=\rho_{l} L A-m_{l, \text { still }}$. 